\title{
Utilization of a Clinical Reminder System to Increase the Incidence of Hepatitis C Screening
}

\author{
Jennifer Cameron* \\ DNP, APRN, FNP-BC Northwestern State University
}

Received: February 1, 2017; Accepted: February 26, 2017; Published: March 4, 2017

*Corresponding author: Jennifer Cameron, DNP, APRN, FNP-BC Northwestern State University, Tel: 318-773-1447. E-mail: jcameronnp@gmail.com

\begin{abstract}
The purpose of this project was to evaluate the use of a clinical reminder system to increase the incidence of patients born between 1945 and 1965 who are offered screening for Hepatitis C Virus (HCV). A clinical reminder form was utilized to prompt primary care providers to offer screening for HCV. Pre-implementation and postimplementation data were collected via retrospective chart review. Data was analyzed to compare patients who were offered testing for HCV pre- and post-implementation of the clinical reminder system. In the pre-implementation data that was collected, 238 patients out of the 600 randomly collected met inclusion criteria of being born between 1945 and 1965 . Of these 238 patients, only four were offered screening for HCV prior to implementation of the project. In the post-implementation data, 248 out of 600 patients met the inclusion criteria of being born between the ages of 1945 and 1965. Of these 248 who met inclusion criteria, 211 were offered screening for HCV. This resulted in $85 \%$ of patients who were offered screening for HCV post-implementation versus $2 \%$ pre-implementation. The results of the study showed that the use of a clinical reminder system was effective in increasing the number of patients who were offered screening for HCV.
\end{abstract}

\section{Introduction}

Hepatitis C virus (HCV) is the most common blood-borne pathogen in the United States (US). According to Gardenier, et al. chronic HCV is estimated to be the cause of $27 \%$ of the cases of liver cirrhosis and $25 \%$ of the cases of hepatocellular carcinoma worldwide [5]. In 2013, the total cost of disability of chronic HCV infection, due to complications of advanced liver disease and cirrhosis, was estimated to be $\$ 6.5$ billion in the US [5]. Approximately $75 \%$ of the HCV disease burden from treatment and disability is found in the population born between 1945 and 1965 [5]. Those born between 1945 and 1965 are referred to as the birth cohort. As the population born between 1945 and 1965 age and the HCV infected population develops complications, it is expected that the cost of HCV disability is expected to rise with a $40 \%$ increase by 2024 with a peak of $\$ 9.1$ billion [5].

According to the Centers for Disease Control (CDC) about 3.5 million Americans are currently living with HCV and roughly half are unaware of their infection [1].The CDC reports that HCV associated mortality is increasing and is now the leading cause of death among all infectious diseases, with a record high 19,659 deaths occurring in 2014 [1]. The CDC also found that in 2013, the number of deaths associated with HCV was greater than the combined number of deaths resulting from 60 other infectious diseases, including Human Immunodeficiency Virus (HIV), pneumococcal disease, and tuberculosis [1]. Because HCV often has few noticeable symptoms, it is expected that the number of new cases of HCV is likely much higher than what is reported. The CDCreports that due to limited screening and underreporting, the number of new cases of HCV is estimated to be closer to 30,000 per year [1].

Efforts to increase screening and detection of HCV, as well as timely referral for treatment is important, so as to decrease the chronic disease of patients with HCV, and to reduce the burden it will place on healthcare resources. According to Gardenier, et al. with chronic HCV infection, the liver parenchyma becomes more fibrotic, and complications such as coagulopathies, reduced protein synthesis, and disrupted homeostatic balance occur, resulting in ascites and volume overload [5]. Chronic HCV progresses to liver cirrhosis and end-stage liver disease, and puts the patient at an increased risk to develop hepatocellular carcinoma. Gardenier, et al. report that liver transplantation needed due to HCV complications is not expected to keep up with the anticipated increased demand, as the number of HCV related complications rise [5].

According to Gardenier, et al. there have been advances in HCV treatment that have resulted in medications that are better tolerated, of shorter duration, and more efficacious. These new treatments are now allowing patients to be cured and decreasing the morbidity and mortality associated with chronic HCV [5].

Patients are not always willing to tell their healthcare provider all their true lifestyle risk factors for HCV and are often asymptomatic, which is why many patients are unaware of their HCV status. A risk-based approach may miss detection of a significant proportion of HCV patients in the birth cohort, because of a lack of patient disclosure or knowledge about prior risk status. In 2012, the CDC recommended one-time screening for all patients born between the years 1945 and 1965 [2]. In 2013, the United States Preventive Services Task Force (USPSTF) also recommended screening for HCV in patients at high-risk for infection and a one-time screening for HCV to patients born between 1945-1965 [13]. 
Research studies have shown that adherence to HCV screening recommendations are low. Linas, et al. report that despite HCV screening recommendations by the CDC and the USPSTF and despite new therapies for HCV only $1 \%-12 \%$ of individuals are screened in the US for HCV infection [6]. A retrospective chart review was performed, in which researchers sought to determine the frequency with which patients are screened for HCV, in order to evaluate the benefits and costs of future interventions to expand HCV screening [6]. The researchers utilized the Kaiser Permanente data repository to investigate HCV antibody screening. In the study 444,594 patients met inclusion criteria of being in the birth cohort. Of those patients, only $14.4 \%$ were ever screened for HCV. This research analysis demonstrated the lack of screening for HCV in the birth cohort, despite recommendations.

Southern, et al. performed a prospective cohort design study in primary care clinics to examine the associations between patient-level, provider-level, and visit-level characteristics and adherence to an HCV screening protocol. Several barriers to adherence with screening recommendations by providers were found, including knowledge base, attitudinal, and external barriers [12]. Examples of these included lack of awareness or familiarity with guidelines, lack of agreement with guidelines, lack of motivation and time, or environmental barriers. In this study, after the providers went through education on the importance of screening, the adherence rate of screening went up only by $36.1 \%$. Adherence with the HCV screening protocol was low, which suggests that attitudinal and external barriers must be addressed in order to obtain maximal adherence to screening recommendations

Ferrante, Winston, Chen, and De La Torre found that primary care providers have insufficient knowledge about screening and counseling for chronic HCV [4]. A cross-sectional mail survey was used and the results indicated that physicians in academic settings were more likely to screen for HCV, providers with over 20 years of experience had lower knowledge of screening for $\mathrm{HCV}$, and providers who were in practice for five or fewer years had more knowledge about screening and increased screening rates. The study concluded that further education for providers regarding HCV screening recommendations is needed.

Ona, Papafragkakis, and Pan performed research that identified barriers to screening [9]. The authors searched electronic databases to identify articles published from 2008 to 2014 on risk-based and birth cohort hepatitis C screening programs in order to better understand the challenge of screening and linkage to care. Patient related barriers included fear of knowing, a low perceived risk by the patient, lack of rapport with the provider, and feeling judged and stigmatized. Healthcare provider related barriers included lack of knowledge regarding screening guidelines. Primary care providers' knowledge deficits may be attributed to limited training, lower caseloads, and consequent lack of confidence to start HCV treatment. The study showed that a large percentage of providers lacked knowledge regarding HCV screening guidelines, which in turn was associated with decreased incidence of HCV screening.
Sidlow and Msaouel performed a retrospective chart review looking at rates of HCV screening [10]. The researchers then designed an HCV testing decision support module that was incorporated into the electronic health record. This HCV testing decision support module triggered an automatic HCV testing order if a patient was eligible for HCV screening and had not been previously tested. The rate of HCV screening for eligible patients born in the birth cohort, and without previously documented HCV test, significantly increased from $11 \%$ to $46 \%$ after implementation of the electronic HCV testing module.

Litwin, et al. performed a serial cross-sectional evaluation of two community-based interventions designed to increase HCV testing in urban primary care clinics [7]. The researchers compared a risk-based approach intervention versus birth cohort screening intervention rates. Both interventions were associated with an increased proportion of patients tested for HCV from $6 \%$ at baseline to $13.1 \%$ during the risk based screener period and 9.9\% during the birth cohort period. The researchers concluded that two simple clinical reminder interventions were associated with significantly increased HCV testing rates.

Southern, et al. performed a study using a cross-sectional design with retrospective electronic medical records to examine the associations between patient demographic and clinical characteristics, testing for anti-HCV, and anti-HCV positivity [11]. The study results concluded that providers test patients with known risk factors for HCV more than they test those in the birth cohort. However, the majority of HCV positive patients identified (73.3\%) were born in the high prevalence birth cohort. These results indicate the importance of testing patients in the birth cohort and not just patients with risk factors for HCV.

Eckman, Talal, Gordon, Schiff, and Sherman performed a Markov state transition model to examine screening of an asymptomatic community-based population in the US and also did a data review of why clinicians and the public are not following HCV screening recommendations [3]. Eckman, et al. reported that data suggest that up to three-quarters of HCV infected patients have yet to be identified [3]. Some reasons providers are not following the recommended guidelines include lack of provider and public awareness, limited time for discussion with patients due to implementation of the many guidelines primary care providers are asked to pursue, chaotic clinical settings, and perceptions of limited efficacy and significant side effect profiles of treatment.

The purpose of this project was to increase the incidence of HCV screening in those born between 1945 and 1965. Most individuals are unaware that they are infected with HCV, because the HCV infected patient usually remains asymptomatic for years. Patients born between 1945 and 1965 have remote risk factors for HCV and, of those infected with HCV most have been infected for over 10 years and are asymptomatic [5]. Prevalence in the 1945 and 1965 age range is four times that of the general population. The increased urgency to screen is because as the birth cohort continues to age, the development of complications is expected to accelerate, leading to increased disease burden. 
The burden of HCV on the health care system will continue to increase, due to the increased complications associated with advanced liver disease and cirrhosis.

Primary care settings offer an important opportunity to incorporate HCV screening into routine visits, in order to diagnose patients with HCV earlier, so that they may seek treatment sooner. When patients are made aware of their HCV status earlier, and are able to seek treatment sooner, this will result in reducing the costs of HCV related disabilities that occurs when patients develop HCV complications.

\section{Methods}

\section{Clinical Question}

Does implementation of a clinical reminder system increase the incidence of HCV screening by primary care providers?

\section{Study Design}

This study is classified as a descriptive study with a retrospective correlational design. The study measured the relationship of using a clinical reminder system to remind providers to screen for HCV, in patients born between 1945 and 1965, by evaluation of testing frequency pre and post implementation. This project was approved by the facility's Institutional Review Board (IRB) as well as the University's IRB. Relevant research was conducted and data was obtained after a thorough literature review. Critique of the literature and data was performed. Based on the literature review, it was felt that screening adherence for HCV in primary care clinics is low and there was sufficient research to pilot the clinical reminder system to remind providers to offer HCV screening in the primary care clinic.

\section{Ethical Considerations}

Prior to project implementation, the project was approved by the IRB at the University and the institutional IRB for the practice site. Because of the retrospective chart review design, this project did not involve substantial risks to the participants. The name of the participants, as well as any other identifying information, was removed prior to data collection. Identifying information was not directly linked to the data or research results in order to ensure minimal or no risk of patient identification. Furthermore, the data was not presented in any way that would compromise the confidentiality of the participants. Analysis of the data did not occur until all portions of the dataset were de-identified.

\section{Setting and Participants}

This study was conducted within a primary care office setting in Louisiana. This clinic population is diverse in race, ethnicity, and socioeconomic status. The clinic providers include six physicians, three nurse practitioners, and one physician assistant. The clinic serves between 500-1000 monthly.

In 2014, in the city where the study was performed, there was a population of 198,242 people with the average age being 35.2 years old. According to the Department of Health and Human Services it is estimated that 70,700 people in Louisiana are infected with HCV [8]. Of these, 50,000 will go on to develop chronic Hepatitis, and 10,000 will develop cirrhosis. It is estimated that 150 Louisiana residents annually die from chronic HCV, and that 3,000 are candidates for costly liver transplants. The number of new HCV cases in Louisiana is increasing. According to the Louisiana Department of Health and Human Services the number of hospitalizations due to HCV increased from 2,337 in 1999 to 7,634 in 2014 [8]. The overall rates of hospitalized Hepatitis C patients were 186.7 per 100,000 males and 105.6 per 100,000 females, with the highest rates seen among those in the 45-64 year-old age range [8].

\section{Instruments}

A clinical reminder form was used in this project to alert the provider of the need for HCV screening. The form was placed on every chart by the front desk staff. The form asked if the patient was born between 1945 and 1965, and a space was provided for the provider to check yes or no. The second question asked if HCV testing was offered, and a space was provided for the provider to check yes or no. The data collection form for the pre and post chart reviews included the patient's gender, age, birth year, insurer, whether the patient was offered HCV screening.

\section{Resources Needed, Project Budget, and Justification}

The clinical reminder form was submitted to a local printer service for copying purposes. The clinical setting was supplied with 1000 copies of the clinical reminder form upon program implementation.

Time requirements of the primary care providers and employees of the clinic were estimated and factored into the cost of implementation. The time requirement for the front desk staff was approximately one to two minutes per chart. The front desk staff was responsible for putting the clinical reminder sheet on all patients' charts that checked in for an appointment during the month of April 2016. The provider then spent three to five minutes of time informing the patient on the recommendations for screening and answering any questions that the patient might have about the test. If the patient agreed for screening, the nurses then took one to two minutes to key the lab test into the system. The estimated noted time this project took per patient was seven to ten minutes, which was donated by the clinic staff. There were minimal additional costs required for the development, dissemination, and evaluation of this scholarly project.

\section{Data Collection and Procedure}

Convenience sampling was used in this project. Charts were reviewed and data collected for patients serviced at the clinic between the dates of February 1, 2016 and April 30, 2016. During this time, patients treated at the clinic prior to implementation of the clinical reminder system, were included in the preimplementation group. This entailed the random selection of 600 patients serviced in the clinic within the time period of February 1, 2016 through February 28, 2018. During the auditing process, gender, age, birth year, insurance status, and whether or not a screening for HCV was offered was collected. 
The sample size of 600 patients was selected, as that was the average number of patients treated at the clinic over the prior three months. No power analysis was needed. Inclusion criteria for the project included being born between the years of 1945 and 1965. Exclusion criteria were if the patient was not born between the years of 1945 and 1965.

A clinical reminder sheet to screen for HCV was placed on all charts for patients treated within the timeframe of April 1-30, 2016. This served as a reminder for the primary care provider to offer HCV screening. The front desk staff was responsible for
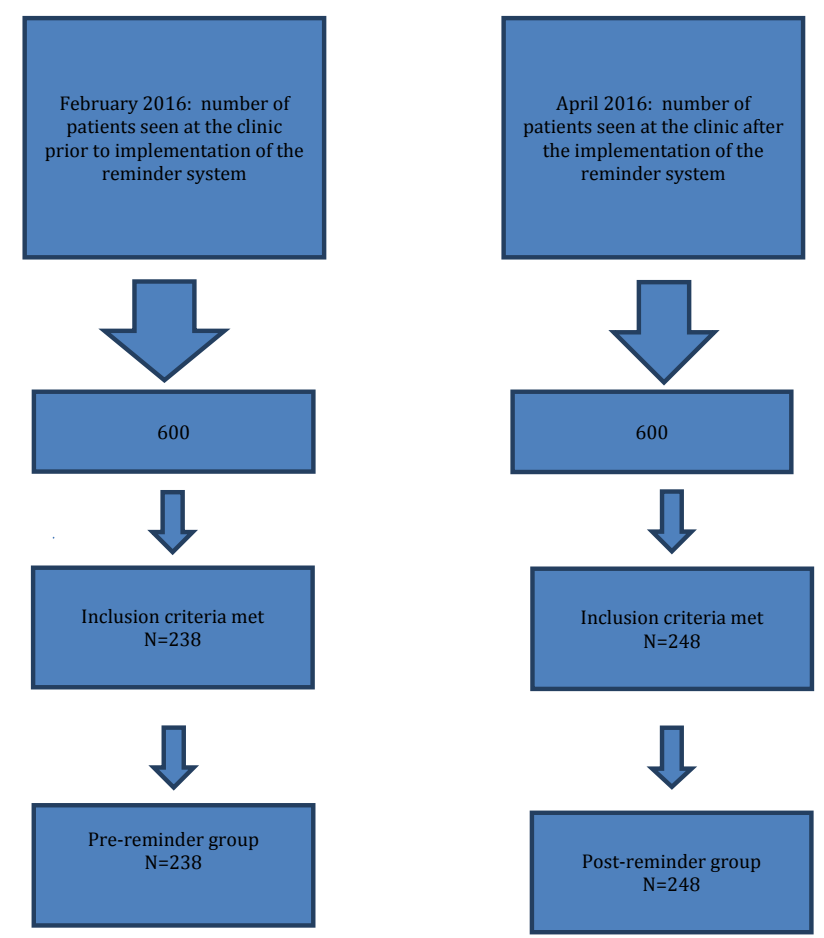

Table 1: Sample Decision Tree

\begin{tabular}{|c|c|c|c|c|}
\hline \multicolumn{1}{|l|}{ Table2. Descriptive Statistics } \\
\hline & Total Subjects & $\begin{array}{c}\text { Average } \\
\text { Age }\end{array}$ & Males & Females \\
\hline $\begin{array}{c}\text { Pre- } \\
\text { Implementation }\end{array}$ & 238 & 61.9 & $76(32 \%)$ & $162(68 \%)$ \\
\hline $\begin{array}{c}\text { Post- } \\
\text { Implementation }\end{array}$ & 248 & 62.1 & $63(25 \%)$ & $185(75 \%)$ \\
\hline
\end{tabular}

\section{Table3. Insurance Statistics}

\begin{tabular}{|c|c|c|c|}
\hline & Total Subjects & $\begin{array}{c}\text { Private } \\
\text { Insurance }\end{array}$ & Medicare \\
\hline $\begin{array}{c}\text { Pre- } \\
\text { Implementation }\end{array}$ & 238 & $130(55 \%)$ & $108(45 \%)$ \\
\hline $\begin{array}{c}\text { Post- } \\
\text { Implementation }\end{array}$ & 248 & $156(63 \%)$ & $92(37 \%)$ \\
\hline
\end{tabular}

Table 4: Differences between Groups for Incidence of HCV Screening offered

\begin{tabular}{|c|c|c|c|c|c|}
\hline $\begin{array}{c}\text { HCV } \\
\text { Screening } \\
\text { Offered }\end{array}$ & $\begin{array}{c}\text { Pre } \\
\text { Implementation }\end{array}$ & $\begin{array}{c}\text { Post } \\
\text { Implementation }\end{array}$ & df & $\mathbf{X}^{\mathbf{2}}$ & $\mathbf{P}$ \\
\hline Yes & $4(2 \%)$ & $211(85 \%)$ & 1 & 351.490 & .000 \\
\hline No & $234(98 \%)$ & $37(15 \%)$ & & & \\
\hline
\end{tabular}

putting the clinical reminder form on each chart when the patient checked in during this time period.

Patients serviced at the clinic during April 1-30 2016, following the implementation of the clinical reminder system, were in the post-implementation group. On May 1, 2016, the retrospective chart review started, the charts of 600 patients treated during this timeframe were retrieved for review. The charts of the selected patients were reviewed in the same way that the pre-implementation charts were reviewed. During the chart review process, demographic data including gender, age, birth year, insurance status, and whether screening for HCV was offered was noted.

Clinic charts were reviewed to collect patient demographic information and to determine whether or not HCV screening was offered. In addition, demographic data was collected via the clinic computer charting system. A retrospective chart review was performed using a data collection tool. All data obtained was then entered into SPSS 22 software for data analysis. Demographic variables were obtained using descriptive and frequency statistics. Group difference between pre and post implementation data was assessed using the chi square test of independence. The level of significance was set at $p<0.001$.

\section{Participant Characteristics}

In the pre-implementation data that was collected, 238 out of 600 patients met inclusion criteria of being born between 1945 and 1965. In the post-implementation data, 248 out of 600 patients met the inclusion criteria of being born between the years of 1945 and 1965. Based on inclusion criteria of being born between 1945 and 1965, there was a total sample size of 486 patients. Characteristics of the pre-implementation patients were an average age of 61.9 years/old, 76 males, and 162 females. Characteristics of the post-implementation patients were an average age of 62.1 years/old, 63 males, and 185 females. Characteristics of the total 486 patients who met inclusion criteria include an average age of 62.17 years old. The payer mix of the group consisted of Aetna, Blue Cross, Cigna, Coventry, Health Plus, Humana, Medicare, Tricare, United, and Vantage Health Plans. There was no Medicaid or self-pay patients included.

\section{Results}

The primary outcome measure of this study was whether or not HCV screening was offered to patients during their clinic visit. A chi square test was used to determine the frequency and percentage of proposed screening. 
In the pre-implementation group of 238 patients, only four were offered screening for HCV prior to implementation of the project. In the post-implementation group of 248 that met inclusion criteria, 211 were offered screening for HCV. This resulted in $85 \%$ of patients being offered screening for HCV post-implementation versus $2 \%$ pre-implementation. A statistically significant difference was seen in the incidence of HCV screening offered between the two groups $\left\{X^{2}(1, N=486)\right.$ $=351.490, \mathrm{p}=.000\}$. This finding also bears clinical significance (Table 5). The results of this project showed that the use of the clinical reminder system to remind providers to screen for HCV was effective. The Iowa Model of Evidence-Based Practice to Promote Quality Care was the framework for this study. After completion of the project, the investigator evaluated if the project met the objectives of the study. Results of the chart review preimplementation revealed there were minimal patients that were offered screening for HCV before implementation of the clinical reminder system. However, with the clinical reminder system, there were a statistically significant number of patients that were offered screening for HCV. Upon evaluation, this project did meet the main objective that showed that the use of a clinical reminder system increased the number of patients that were offered screening for HCV.

\section{Discussion}

This project evaluated the implementation of a clinical reminder system in a primary care setting on the incidence of HCV screening offered. The results were impactful, in that the number of patients born between 1945 and 1965 that were offered screening for HCV significantly increased with the use of the clinical reminder system. Of these patients that were offered screening post-implementation of the clinical reminder system, 5 out of the 211 were positive for HCV, which has resulted in earlier diagnosis. These patients can now seek treatment before becoming symptomatic and the disease has progressed, which decreases total healthcare costs and reduces morbidity and mortality from HCV.

Targeted HCV screening in the primary care setting is important for the identification of patients who are infected with HCV that might not otherwise be diagnosed. HCV screening of patients born in the 1945-1965 birth cohort is an important part of the screening performed in primary care, because HCV causes negative health outcomes and can be diagnosed before symptoms appear. Testing for HCV is readily available, minimally invasive, and reliable. Benefits of testing include limiting disease progression and facilitating early access to treatments that can save significant life years; and testing is cost effective. As recommendations by the CDC and USPSTF are both still new, more literature is needed looking at the cost and benefit ratio of screening of those born between 1945-1965. As HCV causes a huge burden on the healthcare system, it is important to increase awareness of the HCV screening recommendations. Advanced Practice Registered Nurses can play a key role in educating other providers and patients on the importance of increased screening for HCV.

\section{References}

1. Centers for Disease Control and Prevention. Hepatitis C kills more Americans than any other infectious disease. 2016. doi: 10.1371/ journal.pone.0058975

2. Centers for Disease Control and Prevention. Recommendations for the identification of chronic hepatitis $\mathrm{C}$ virus infection among persons born during 1945-1965. 2012.

3. Eckman MH, Talal AH, Gordon SC, Schiff E, Sherman KE. Costeffectiveness of screening for chronic hepatitis $\mathrm{C}$ infection in the United States. Clinical Infectious Diseases. 2013;56(10),1382-1393. doi: $10.1093 / \mathrm{cid} / \mathrm{cit} 069$

4. Ferrante JM, Winston DG, Chen PH, de la Torre AN. Family physicians' knowledge and screening of chronic hepatitis and liver cancer. Health Services Research. 2008;40(5),345-351.

5. Gardenier D, Kwong J, Olson MC, Epstein R. Epidemiology, screening, and pretreatment evaluation of the patient with chronic hepatitis $\mathrm{C}$ infection. The Journal for Nurse Practitioners. 2015;11(1),109-115. doi: 10.106/j.nurpra.2014.10.015

6. Linas BP, Hu H, Barter DM, Horberg M. Hepatitis C screening trends in a large integrated health system. The American Journal of Medicine. 2014;127(5),398-405. doi: 10.1016/j.amjmed.2014.01.012

7. Litwin AH, Smith BD, Drainoni ML, McKee D, Gifford AL, et al. Primary care-based interventions are associated with increases in hepatitis $\mathrm{C}$ virus testing for patients at risk. Digestive and Liver Disease. 2012;44(6),497-503. doi: 10.1016/j.dld.2011.12.014

8. Louisiana Department of Health and Human Services .Hepatitis C annual report. 2014

9. Mel A Ona, Haris Papafragkakis, Calvin Q Pan. Hepatitis C screening in the United States: Current models and challenges. American Journal of Digestive Diseases. 2015;2(1),29-40. doi: 10.73626/0003-4819156-4-201202210-00378

10. Sidlow R, Msaouel P. Improving hepatitis $C$ virus screening rates in primary care: A targeted intervention using the electronic health record. Journal for Healthcare Quality. 2015;37(5),319-323.doi: 10.1097/JHQ.00000000000010

11. Southern WN, Drainoni ML, Smith BD, Christiansen CL, McKee D, et al. Hepatitis $C$ testing practices and prevalence in a high-risk urban ambulatory care setting. Journal of Viral Hepatitis. 2011;18(7), 471481. doi: 10.1111/j.1365-2893.2010.01327.x

12. Southern WN, Drainoni ML, Smith BD, Koppelman E, McKee MD, et al. Physician nonadherence with a hepatitis C screening program. Quality Management in Health Care. 2014;23(1),1-9. doi: 10.10197/ QMH.0000000000007

13. United States Preventive Services Task Force. Recommendations for primary care practice. 2015. 